\title{
Spatiotemporal behavior of nuclear cyclophilin B indicates a role in RNA transcription
}

\author{
BIRGER DIERIKS $^{1}$ and PATRICK VAN OOSTVELDT ${ }^{1,2}$ \\ ${ }^{1}$ Department of Molecular Biotechnology, and ${ }^{2}$ Center for Nano- and Biophotonics \\ (NB-Photonics), Ghent University, Ghent, Belgium
}

Received November 18, 2011; Accepted December 22, 2011

DOI: $10.3892 / \mathrm{ijmm} .2012 .937$

\begin{abstract}
Cyclophilin B (CypB) is an ubiquitously expressed protein, which performs several intra- and extracellular functions. Despite its abundant use as a household protein, little is known about its exact cellular localization and dynamics. In the present study we show that endogenous CypB localizes in one of two distinct compartments, either within the endoplasmic reticulum (ER) or inside the nucleus, accumulating in the fibrillar centers of the nucleoli. By means of a genetic deletion screen, we identified a minimal nucleolar localization signal for efficient relocation to the nucleoli. Within the fibrillar centers, CypB colocalized with RNA polymerase, upstream binding factor-1 (UBF), fibrillarin and dyskerin (DCK1). Even after chemical disruption of the nucleoli, a strong interaction with these proteins remained. Using live cell imaging, we showed a persistent colocalization of $\mathrm{CypB}$ with proteins involved in the ribosome biogenesis during the transcriptionally more active phases of the cell cycle. Supported by in silico data, our observations suggest that $\mathrm{CypB}$ interacts with these proteins and is involved in ribosome biogenesis and RNA transcription.
\end{abstract}

\section{Introduction}

Cyclophilins are ubiquitously distributed intracellular proteins first identified as cellular binding proteins for the potent immunosuppressive drug, cyclosporin A (CsA). Also known as peptidyl-prolyl cis-trans isomerases, they catalyze the slow cistrans isomerization of proline peptide bonds in oligopeptides and accelerate folding of several proteins. Cyclophilins are highly conserved, suggesting a crucial role in the function of living cells (1). The cyclophilins are closely related but mainly differ in signal sequences located at the $\mathrm{N}$ - and C-termini. On the N-terminus of cyclophilin $\mathrm{B}(\mathrm{CypB})$ an endoplasmic reticulum (ER)-localization signal of 33 amino acids is located,

Correspondence to: Dr Birger Dieriks, Department of Molecular Biotechnology, Ghent University, Coupure Links 653, 9000 Ghent, Belgium

E-mail: birger.dieriks@gmail.com

Key words: cyclophilin B, nucleolus, live cell imaging, endoplasmic reticulum which is usually not present in the mature protein. This is followed by a nuclear translocation motif (DEKKKGPKV) (2). On the C-terminal end, the VEKPFAIAKE sequence has been described as a signal for retention in intracellular vesicles (3). This sequence allows CypB to be present in both the cytoplasm and the nucleus but also to become secreted. A truncated form of $\mathrm{CypB}$, lacking the C-terminal AIAKE sequence (4), is found in several biological fluids such as human milk and serum (5). This secretion is accelerated after CsA incubation (6). Although CypB contains an ER localization signal, when examined biochemically the majority is found to reside in the cell nucleus, with lower quantities in the ER compartment (7). In the ER, CypB functions as a chaperone protein and interacts with other chaperone proteins, such as protein disulfide isomerase (PDI). Complexation of CypB with CsA completely inhibits the chaperone effect of PDI (8). CypB is also a critical factor for the efficient replication of the hepatitis $\mathrm{C}$ genome. Both NS5B and the hepatitis C virion (HCV) RNA are mainly found on the cytoplasmic surface of the ER, where they colocalize with a fraction of $\mathrm{CypB}$. CypB interacts directly with the HCV RNA polymerase NS5B to stimulate its RNA binding activity (9). CsA is able to reduce the expression of $\mathrm{HCV}$ encoded proteins to undetectable levels (10). Recent results indicate that recombinant $\mathrm{CypB}$ purified from insect cells directly stimulates NS5B-catalyzed RNA synthesis through an increase in productive RNA binding (11). In addition to its functions in the ER, CypB has various functions within the nucleus, among which transcriptional activation $(12,13)$. To provide new insight in the different functions of CypB we have performed a genetic deletion screen, whereby we monitored the protein's spatiotemporal location in human fibroblasts and HeLa cells. We discovered that the nuclear $\mathrm{CypB}$ fraction accumulates in the fibrillar centers (FCs) of the nucleoli, where it colocalizes with several proteins involved in ribosome biogenesis. The potential implications for $\mathrm{CypB}$ function in RNA and ribosome metabolism are discussed.

\section{Materials and methods}

Cell culture. Human dermal fibroblasts isolated from normal juvenile foreskin (HDF) (PromoCell) and HeLa cells were grown in advanced DMEM supplemented with $2 \%$ FCS, $1 \%$ L-glutamine, streptomycin-penicillin (Life Technology) at $37^{\circ} \mathrm{C}$ in a humidified incubator containing $5 \% \mathrm{CO}_{2}$. HDF cells were 
Table I. Primer sequences used for construction of CypB constructs.

Name

Primer sequence $5^{\prime} \rightarrow 3^{\prime}$

attb1 (sequencing)

attb2 (sequencing)

4 forward

4 reverse

5 reverse

6 forward

7 reverse

8 reverse

9 forward

9 reverse

10 reverse

11 forward

12 forward

13 forward

14 forward

\begin{abstract}
ACAAGTTTGTACAAAAAAGCAGGCT
ACCACTTTGTACAAGAAAGCTGGGT

GGGGACAAGTTTGTACAAAAAAGCAGGCTTCACCATGCTGCGCCTCTCCGAACG

GGGGACCACTTTGTACAAGAAAGCTGGGTTCTACTCCTTGGCGATGGCAA

GGGGACCACTTTGTACAAGAAAGCTGGGTGCTCCTTGGCGATGGCAAAGG

GGGGACAAGTTTGTACAAAAAAGCAGGCTTCACCATGGATGAGAAGAAGAA

GGGGACCACTTTGTACAAGAAAGCTGGGTTCTAAAAGGGCTTCTCCACCT

GGGGACCACTTTGTACAAGAAAGCTGGGTGAAAGGGCTTCTCCACCTCGA

GGGGACAAGTTTGTACAAAAAAGCAGGCTTCACCATGTATTTTGACCTACGAAT

GGGGACCACTTTGTACAAGAAAGCTGGGTTCTACTTGCCGCAGTCTGCGA

GGGGACCACTTTGTACAAGAAAGCTGGGTGCTTGCCGCAGTCTGCGATGA

GGGGACAAGTTTGTACAAAAAAGCAGGCTTCACCATGAAGAAGGGGCCCAAAGTC

GGGGACAAGTTTGTACAAAAAAGCAGGCTTCACCATGAAGGGGCCCAAAGTCAC

GGGGACAAGTTTGTACAAAAAAGCAGGCTTCACCATGGGGCCCAAAGTCACCGT

GGGGACAAGTTTGTACAAAAAAGCAGGCTTCACCATGGTCACCGTCAAGGTGTAT
\end{abstract}

harvested by trypsinization, counted and replated at the appropriate densities on 18-mm diameter glass coverslips (VWR International).

Immunofluorescence. Cells were grown on glass coverslips up to a confluency rate of about $70 \%$ after which they were rinsed with PBS and fixed in $2 \%(\mathrm{v} / \mathrm{v})$ paraformaldehyde (VWR International) in PBS for $15 \mathrm{~min}$ at ambient temperature. Slides were washed in PBS for $3 \times 5$ min and subsequently permeabilized in $0.5 \%$ Triton X-100 solution for $5 \mathrm{~min}$ on ice. In replication labeling experiments, $20 \mu \mathrm{M}$ BrdU (Sigma-Aldrich) was added to the cell culture medium $40 \mathrm{~min}$ prior to fixation. In order to reveal the BrdU epitopes for immunofluorescence the cells were exposed to $0.1 \mathrm{M} \mathrm{HCl}$ for $10 \mathrm{~min}$. Subsequently, cells were washed with PBS, blocked with 50\% FCS in PBS for $20 \mathrm{~min}$ at room temperature and incubated for $1 \mathrm{~h}$ at room temperature with one or with a combination of primary antibodies (rabbit anti-CypB, 1:600, ab3565; mouse anti-Coilin, 1:2,000, ab11822; mouse anti-PDI, 1:1,000, ab2792 (Abcam); mouse anti-PML, 1:1,000, sc-966; rabbit anti-fibrillarin, 1:50, sc-25397; mouse anti-RNA-POL, 1:250, sc-21751; mouse anti-upstream binding factor-1 (UBF), 1:50, sc-13125, (Santa Cruz Biotechnology, Inc.); mouse anti-BrdU, 1:500, A21300; mouse anti-golgin-97, 1:2,000, A21270 (Invitrogen). Cells were subsequently washed in PBS for $3 \times 5$ min and incubated with fluorescently labeled secondary antibodies for $45 \mathrm{~min}$ (goat anti-mouse Alexa fluor 488, 1:600, A10667; goat antimouse Alexa fluor 555, 1:600, A21422; goat anti-rabbit Alexa fluor 488, 1:600, A11070 (Invitrogen); goat anti-rabbit Hylite 555, 1:600, 28172 (Anaspec). The cells were washed 5 times with PBS, stained with DAPI (10 ng/ml; Life Technologies) or propidium iodide (PI, $1 \mu \mathrm{g} / \mathrm{ml}$; Life Technologies) for $90 \mathrm{sec}$, washed and mounted with Vectashield reagent (Vector Laboratories) on glass slides and sealed with nail polish.

cDNA synthesis, GFP constructs, transfection. Total-RNA from HDF cells was isolated from T75 culture flasks with TRI
Reagent (Applied Biosystems) according to manufacturer's instructions. First-strand cDNA was prepared from a sample of $2 \mu \mathrm{g}$ total-RNA in a volume of $40 \mu \mathrm{l}$ with the SuperScript reverse transcriptase II first strand cDNA synthesis kit according to the manufacturer's instructions (Life Technologies).

Different CypB-GFP constructs were designed and constructed according to the manufacturer's instructions (Gateway Technology, Life Technologies). The primers and primer combinations are described in Tables I and II. CypBsequences were picked up from cDNA derived from HDF cells and inserted into pDONR 221 clones (entry vector). Gateway recombination sites were added in a two-step PCR reaction. The plasmids were purified with the nucleobond plasmid miniprep system, according to the manufacturer's instructions (MachereyNagel). Vectors were subsequently sequenced (Addgene). CypB gene sequences were cloned into pcDNA-Dest53 and pcDNADest47 with GFP at the 3 ' or 5' end, respectively. For live cell experiments, cells were grown on glass bottom dishes (MatTek Corporation). The obtained constructs were introduced in HDF and HeLa cells using Lipofectamine 2000 (Life Technologies). Visualization and additional experiments were performed $24 \mathrm{~h}$ after transfection. Care was taken to perform and visualize all transfections under identical conditions of illumination and environmental control.

Drug treatments. For ATP depletion, $10 \mathrm{mM}$ sodium azide in combination with $50 \mathrm{mM}$ 2-deoxyglucose (2-DG; Sigma-Aldrich) was added to the medium during imaging. CsA (Sigma-Aldrich) is a functional inhibitor of $\mathrm{CypB}$ and was used at $2.5 \mu \mathrm{M}$. Cycloheximide (CHX; $100 \mu \mathrm{g} / \mathrm{ml}$ Sigma-Aldrich) and the adenosine analog 5,6-dichloro- $\beta$-D-ribofuranosylbenzimidazole (DRB, $50 \mu \mathrm{g} / \mathrm{ml}$; Sigma-Aldrich) were used to block translation or RNA polymerase II-dependent transcription, respectively.

Fluorescence mosaic and confocal microscopy. Fluorescence recordings were made on an automated Nikon TE2000E epifluorescence microscope, equipped with an EM-CCD 
Table II. Primer combinations used for construction of CypB constructs.

\begin{tabular}{lcc}
\hline Construct name & \multicolumn{1}{c}{ N-term GFP } & C-term GFP \\
\hline CypB-Full & 4 forward-4 reverse & 4 forward-5 reverse \\
CypB-Nucl & 6 forward-4 reverse & 6 forward-5 reverse \\
CypB-Nucl2 & 6 forward-7 reverse & 6 forward-8 reverse \\
CypB-Golgi & 4 forward-7 reverse & 4 forward- 8 reverse \\
CypB-Iso & 9 forward-9 reverse & 9 forward-10 reverse \\
CypB-Del1 & 11 forward-4 reverse & 11 forward-5 reverse \\
CypB-Del2 & 12 forward-4 reverse & 12 forward-5 reverse \\
CypB-Del3 & 13 forward-4 reverse & 13 forward-5 reverse \\
CypB-Del4 & 14 forward-4 reverse & 14 forward-5 reverse \\
\hline
\end{tabular}

camera (Andor iXon+). Acquisition was performed with the Andor IQ software. The metal halide lamp was attenuated by an ND4 filter to minimize photobleaching. DAPI, Alexa-488 or GFP and Alexa-555 were respectively excited with bandpass excitation filters $377 / 50,485 / 20,562 / 40$, separated by a triple dichroic type 436/514/604 and detected through emission filters 457/50, 528/38,624/40, respectively. For confocal recordings a Nikon TE Eclipse 2000 confocal microscope was used, equipped with a Plan Apo 60x/1.4 Oil objective.

\section{Results}

Endogenous CypB shows a nuclear and a cytoplasmic localization pattern. To map the basal localization pattern of endogenous $\mathrm{CypB}$, immunostaining was performed on human primary fibroblasts (HDF) and HeLa cells. Two major localization patterns could be distinguished: a cytoplasmic and a nuclear phenotype. In some cases, CypB was observed throughout the complete culture in small foci scattered throughout the cytoplasm with no significant CypB staining in the nucleus (cytoplasmic phenotype) (Fig. 1A). Alternatively, cells were observed, which only showed a low diffuse background signal in the cytoplasm, but a pronounced CypB staining of the nucleus and prominent accumulation in the nucleoli (nuclear phenotype). Replication labeling showed that this localization was cell cycle-dependent (Fig. 1B). This cell cycle-dependent pattern was observed in both HeLa cells and HDF. In G1, multiple small spots (submicron size) of CypB were evenly distributed throughout the nucleus. Throughout the S-phase the nucleolar localization pattern became more apparent and persisted in the G2 phase (Fig. 1B). In mitotic cells, CypB staining delineated the condensed chromosomes completely only to return to a diffuse pan-nuclear staining in G1. Occasionally (10\%) cells with clear nuclear staining also showed cytoplasmic foci, resembling those observed in the cytoplasmic phenotype. This variability in the localization pattern could not be explained by varying culture conditions like medium composition, cell density or fixation.

Differential western blot analysis confirmed that $\mathrm{CypB}$ was present in both the nuclear and the cytoplasmic fractions (Fig. 1C). In addition, cells constitutively secreted CypB, as demonstrated by a gradual increase of extracellular CypB with culture time. CypB was clearly visible in the superna-
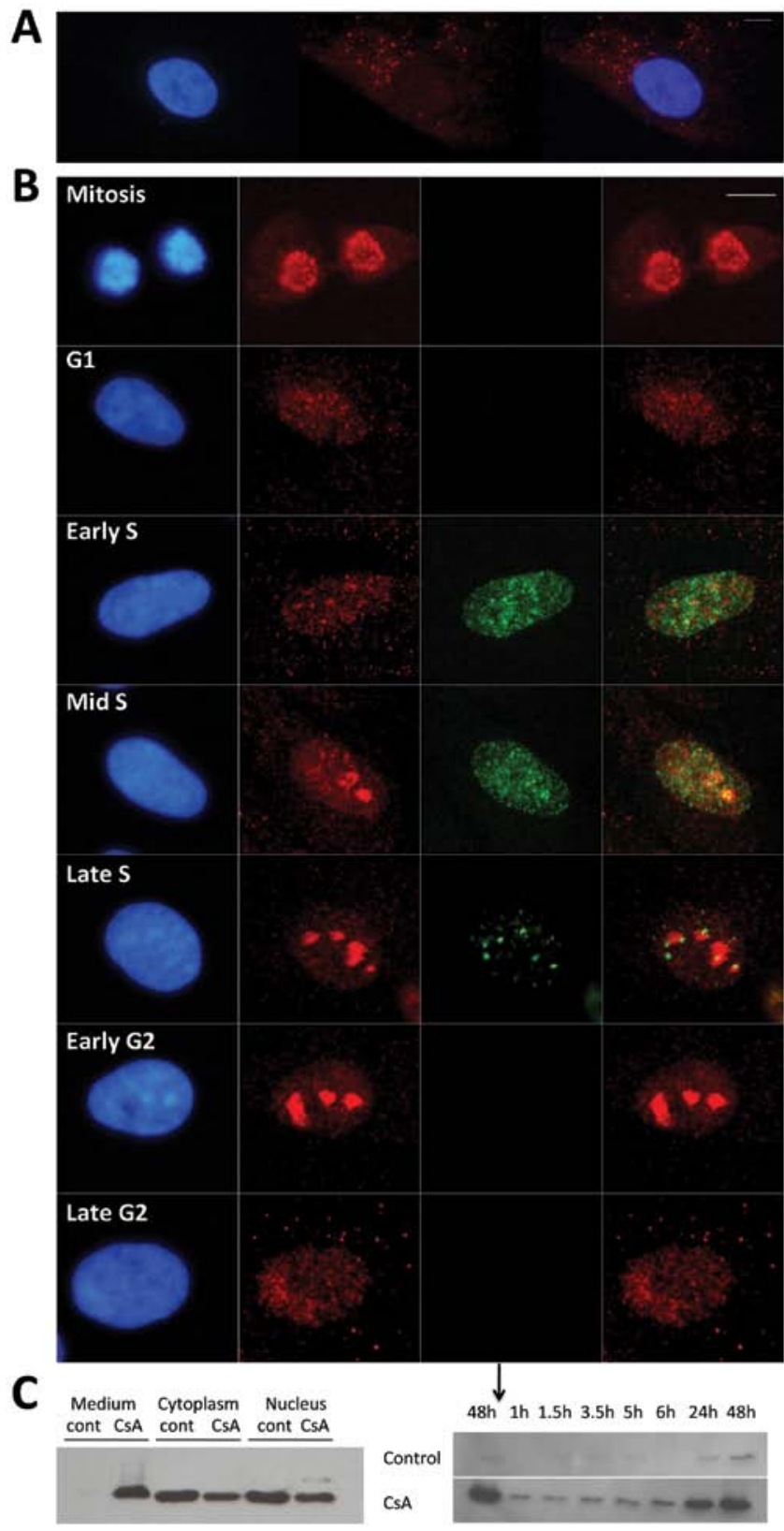

Figure 1. Immunofluorescence staining of primary fibroblasts cell cultures (A) Primary fibroblast cells with a cytoplasmic staining pattern, with the nucleus devoid of CypB. (B) Primary fibroblasts with a distinct nuclear expression pattern throughout the cell cycle. From left to right: DAPI staining of the nucleus (blue), CypB (red), BrdU as a specific S-phase marker (green) and the merged image. During mitosis $\mathrm{CypB}$ is present around the chromosomes. Cells in the G2 phase no longer display a visible BrdU pattern but CypB still remains in the nucleoli. When cells progress in late G2 the strong nucleolar localization is replaced by a generalized staining of the nucleus. Some of these cells display intense spots within the cytoplasm. Scale bar, $10 \mu \mathrm{m}$. (C) Inter and extracellular expression levels of CypB. Analysis of nuclear, cytoplasmic and secreted fraction of HDF cells after $24 \mathrm{~h}$. A small increase in the molecular weight (MW) is observed when comparing the MW of secreted CypB to the endogenous form. CypB secretion in the supernatant steadily increases. When CsA is added this increase is significantly higher. After $48 \mathrm{~h}$ fresh medium with or without CsA was added (arrow).

tant of HDF cells after $24 \mathrm{~h}$ of cell culture. CsA is known to induce CypB secretion (14). Indeed, addition of $2.5 \mu \mathrm{M}$ CsA immediately after plating, significantly increased CypB secretion while reducing both cytoplasmic and nuclear СypB 

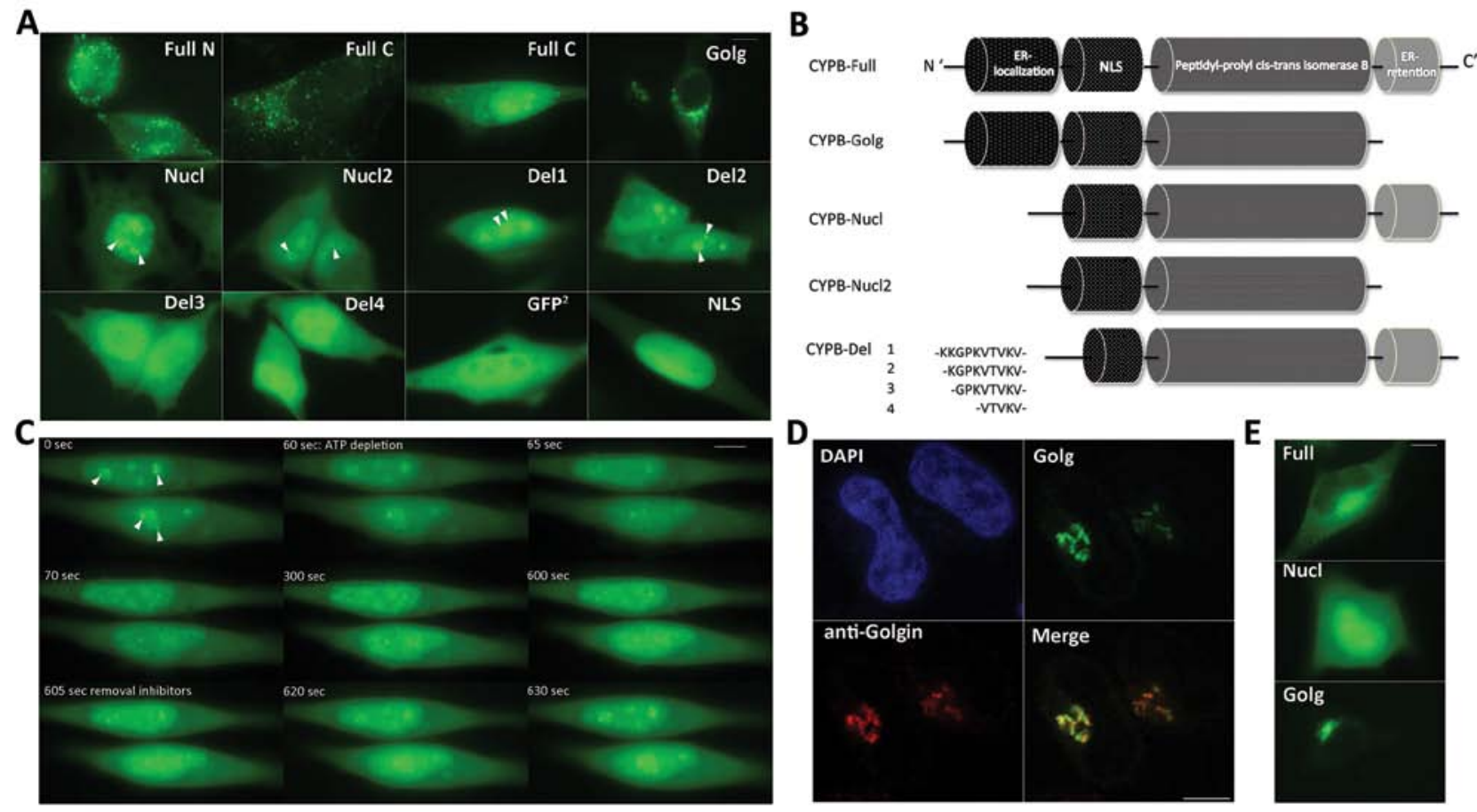

Figure 2. Transient expression patterns of the various CypB-GFP constructs and the respective controls. (A) For each transfection a representative image of the dominant phenotype is shown. The CypB-Full C-terminus has two different expression patterns either in spots scattered throughout the cytoplasm or localized in the nucleus; with the exception of CypB-Full all the constructs that are displayed have an N-terminal GFP; arrows indicate intense spots mostly residing within the nucleoli; controls used are GFP ${ }^{2}$, which are two fused GFP's, and secondly a known NLS localization signal. (B) Schematical overview of the different CypB vectors. (C) Subcellular distribution of HeLa cells transfected with CypB-Nucl after ATP depletion. Sixty seconds later NaN ${ }_{3}$ and 2-DG were added together to inhibit energy-dependent processes. Addition of the inhibitors leads to an immediate evacuation of CypB located in the nucleoli. Only the intense spots remain. After $600 \mathrm{sec}$ the inhibitors were removed and the initial phenotype returned. (D) Immunofluorescent staining of transfected HeLa cells with CypB-Golgi and with anti-golgin-97. CypB-Golgi and golgin-97 stain similar regions within the cell. (E) Treatment of transfected cells with 2,5 $\mu \mathrm{M} \mathrm{CsA}$ CsA treatment had similar effects on CypB-Nucl and CypB-Nucl2; For the control constructs no detectable effects for CsA were observed; Scale bar, $10 \mu \mathrm{m}$.

concentrations. Strikingly, secreted CypB showed a higher MW (approximately, $0.5 \mathrm{kDa}$ ) suggesting post-translational modification before secretion.

Deletion screen reveals different localization patterns. To study the spatiotemporal localization of CypB in living cells, we engineered a full-length fusion construct for $\mathrm{CypB}$ coupled to GFP. Both an N-terminal and a C-terminal construct were designed and expressed in HDF or HeLa cells. In 95\% of the cells, expression of the C-terminal full length CypB (CypBFull-GFP) resulted in a cytoplasmic pattern of fluorescent spots and no substantial signal in the nucleus; a phenotype identical to the endogenous cytoplasmic pattern. CypBFull-GFP containing spots moved fast in a directed manner within the cytoplasm and colocalized with PDI (Fig. 3F). In a small subset (5\%) of cells CypB-Full-GFP also accumulated in the nucleus (Fig. 2A). Expression of N-terminal full length CypB (GFP-CypB-Full) did not produce a functional CypB-GFP construct.

Based on previous information we identified an ER-localization sequence (AA1-33, ELS) and an ER retention signal (AIAKE, ERS). Hence, to correlate CypB localization with its spatiotemporal processing and targeting, we constructed different CypB-mutants by directed deletion of (presumed) signal sequences of the full-length protein. These constructs allowed us to study cells that consistently expressed CypB in one of the different subcompartments (Fig. 2A). For every deletion, a C- and an N-terminal construct were made, but unless explicitly stated, no differences were observed in localization. Removal of the ERS (CypB-Golgi) significantly reduced the ER localization of $\mathrm{CypB}$. Instead of small cytoplasmic spots $(<1 \mu \mathrm{m})$, a larger $(>2 \mu \mathrm{m})$ uniform region of CypB was observed close to the nucleus. Colocalization with anti-golgin by confocal microscopy revealed that these structures corresponded to the Golgi apparatus (Fig. 2D). However, when the ELS was deleted (CypB-Nucl) a predominant nuclear staining was observed, with specific enrichment in the FCs of the nucleoli. This was also confirmed by immunostaining for fibrillarin, RNA POL and UBF (Fig. 3C) and an absence of PI staining (Fig. 3E). An identical pattern was observed when both ER-localization and ER-retention sequence were removed (CypB-Nucl2) (Fig. 2A). To verify that this localization was not caused by overexpression of a GFP construct, we made use of two controls. As a first control, a double GFP $\left(\mathrm{GFP}^{2}\right)$ construct was used as the size of the translation product approximated the size of CypB-Nucl. GFP ${ }^{2}$ showed a homogeneous pancellular distribution except for the nucleoli where there was no signal. A second control was an NLS-EYFP construct (GGPKKKRKV) (15), which uniformly stained the nucleus with no specific nucleolar accumulation. These experiments emphasize the necessity of a NuLS signal for directed localization to the nucleoli (Fig. 2A).

Removal of the first 33 amino acids (ELS) of the CypB was sufficient for nuclear localization. DEKKKGPKVTVKV 

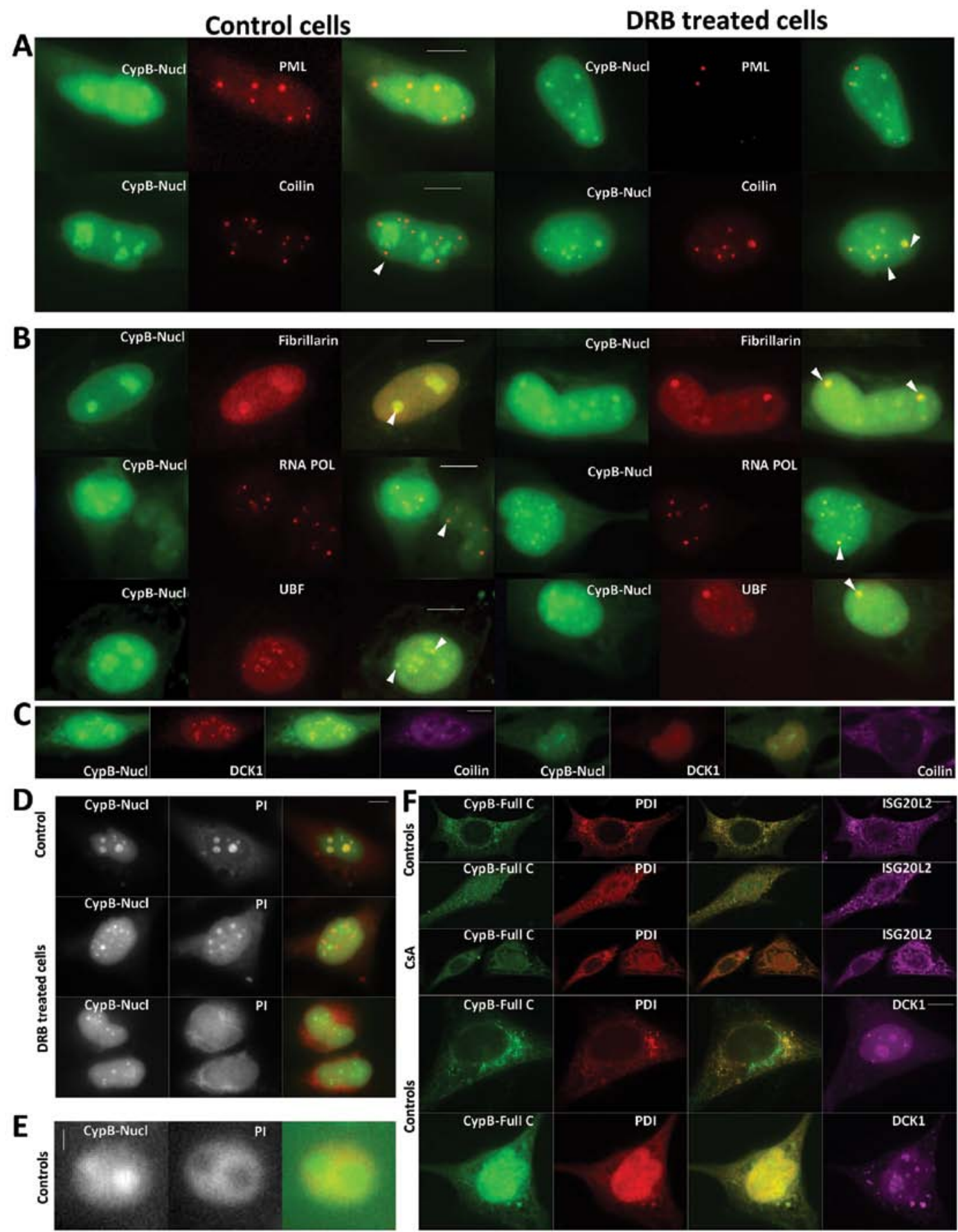

Figure 3. (A) Subcellular distribution of HeLa cells transfected with CypB-Nucl and the relationship with nuclear bodies. For each staining representative images of both control and DRB-treated $(400 \mathrm{~min})$ cells are shown. No colocalization of CypB-Nucl and PML was observed; some of the Cajal bodies colocalized with CypB-Nucl (arrows). After treatment with DRB it appeared that the interaction with CypB-Nucl increased. It encapsulated the larger CypB spots that were still present within the cells (arrows). (B) Fibrillarin associated with CypB-Nucl in the nucleoli and to a higher degree in the fibrillar centers (FCs). After DRB treatment, in the regions with no staining for fibrillarin, CypB staining was more pronounced. UBF and RNA POL both colocalized strongly in the FCs of the nucleoli. After treatment with DRB, RNA-POL retains contact with CypB. It encapsulated the larger CypB spots that were still present within the cells (arrows); UBF on the other hand only colocalized with some of the intensely staining CypB-Nucl spots. (C) For DCK1 a general staining of the nucleus and an intense staining of the nucleoli and the Cajal bodies is observed. DRB treatment causes DCK1 to dissipate throughout the nucleus and to loose most of the colocalization with CypB and the Cajal bodies. (D) DRB treatment effects the chromosomal architecture. Without DRB CypB-Nucl colocalized with the nucleoli. DRB disrupted nuclear architecture and CypB and PI no longer colocalized. In some cells PI did not stain any structures. (E) Magnification of x10 of nucleolus in non-treated HeLa. The intense CypB spots clearly coincide with the darker stained regions in the nucleolus. (F) Immunofluorescent staining of transfected HeLa's with CypB-Full C-terminal expression. Large portion of CypB-Full C colocalized with PDI and ISG20L2. Two types of staining patterns were observed. Either an ER localized staining or a nuclear staining with ER staining (5\%). The relocalization of CypB-Full C to the nucleus affected both PDI as DCK1 and to a smaller extend ISG20L2. (A-D and F) Scale bar, $10 \mu \mathrm{m}$; (E) $1 \mu \mathrm{m}$.

was described as an NLS signal (2). However, until now it was unclear which of the amino acids are essential for this local- ization and especially, which are responsible for the nucleolar localization. To clarify this we made different deletion mutants 
Table III. List of genes localized in the nucleoli and regulated by CypB.

\begin{tabular}{|c|c|c|c|}
\hline Symbol & Description & Fold-change & UniGene \\
\hline HSPA8 & Heat shock $70 \mathrm{kDa}$ protein 8 & 2.3439 & Hs.702021 \\
\hline MORF4L1 & Mortality factor 4 like 1 & 2.0256 & Hs.374503 \\
\hline CCDC72 & Voiled-coil domain containing 72 & 1.7289 & Hs.356440 \\
\hline DKC1 & Fyskerin, H/ACA ribonucleoprotein complex subunit 4 & 1.7214 & Hs.4747 \\
\hline HIST1H2AE & Histone $\mathrm{H} 2 \mathrm{~A}$ type $1-\mathrm{B} / \mathrm{E}$ & 1.6696 & Hs. 121017 \\
\hline BUB3 & Mitotic checkpoint protein BUB3 & 1.616 & Hs.418533 \\
\hline LOC729973 & Hypothetical LOC729973 & 1.6014 & NA \\
\hline ISG20L2 & Interferon stimulated exonuclease gene $20 \mathrm{kDa}$-like 2 & 1.5736 & Hs.301904 \\
\hline RPL14 & $60 \mathrm{~S}$ ribosomal protein $\mathrm{L} 14$ & 1.5623 & Hs. 446522 \\
\hline VDAC1 & Voltage-dependent anion-selective channel protein 1 & 1.5566 & Hs. 519320 \\
\hline KPNA2 & Karyopherin subunit $\alpha-2$, Importin subunit $\alpha-2$ & 1.5392 & Hs.594238 \\
\hline MANF & Mesencephalic astrocyte-derived neurotrophic factor & 1.5101 & Hs.436446 \\
\hline SF3A3 & Splicing factor 3a, subunit 3,60 kDa & -1.5511 & Hs.77897 \\
\hline TAF15 & TATA-binding protein-associated factor $2 \mathrm{~N}$, RNA-binding protein 56 & -1.6025 & Hs.402752 \\
\hline HDAC1 & Histone deacetylase 1 & -1.6601 & Hs.88556 \\
\hline SHMT2 & Serine hydroxymethyltransferase (mitochondrial) & -1.7073 & Hs.75069 \\
\hline TOP2A & Topoisomerase (DNA) II $\alpha$ & -1.7159 & Hs. 156346 \\
\hline EEF1A2 & Elongation factor $1-\alpha 2$ & -1.7196 & Hs.433839 \\
\hline SEPT11 & Septin 11 & -2.0185 & Hs.128199 \\
\hline SUPT16H & FACT complex subunit SPT16 & -2.0458 & Hs. 213724 \\
\hline HSPA5 & $78 \mathrm{kDa}$ glucose-regulated protein & -2.0521 & Hs.605502 \\
\hline S100A4 & S100 calcium binding protein A4 & -2.917 & Hs. 654444 \\
\hline CYPB & Cyclophilin B & -13.127 & Hs.434937 \\
\hline
\end{tabular}

by selectively eroding the presumed NLS (Fig. 2B) and found KGPKVTVKV to be the minimum sequence required for CypB to relocate the nucleoli and accumulate in the FCs (Fig. 2A).

Cyclosporin A induces transient as well as permanent translocation of different CypB proteins. Addition of CsA induced some notable changes in localization patterns and expression. In all constructs the overall intensity decreased, as observed in HDF cells. For CypB-Full a decrease in intensity of the CypB spots was seen. Upon removal of CsA, the phenotype was completely restored. Addition of CsA to CypB-Golgi seemed to accelerate the decrease of $\mathrm{CypB}$ spots in the cytoplasm but did not affect the CypB within the Golgi (Fig. 2E). For CypBNucl, addition of CsA ( $4 \mathrm{~h}$ ) caused a decrease in staining of the nucleoli. Especially the signals in the fibrillar centers vanished completely. Intriguingly, removal of CsA by washing and renewal of the culture medium only partially restored the phenotype in a small number of cells (5\%). Even $24 \mathrm{~h}$ after medium exchange CypB-Nucl was still not present into the fibrillar centers.

Nuclear CypB is actively recruited to fibrillar centers and associates with Cajal bodies upon disruption of nucleoli. When ATP synthesis was blocked using $\mathrm{NaN}_{3}$, the majority of CypB-Nucl proteins moved out of the nucleoli. Upon removal of the inhibitor the nucleolar phenotype was restored (Fig. 2C). For comparison, $\mathrm{GFP}^{2}$ distribution was unaffected by $\mathrm{NaN}_{3}$ and 2-DG (data not shown). As the nucleoli still remained, this suggests that CypB-Nucl is directed to the nucleoli in an ATP-dependent fashion. However, part of the CypB-Nucl remained sequestered in intense spots (presumably the fibrillar centers) upon ATP depletion. We next dispersed the nucleoli, including the fibrillar centers, by using the adenosine analog DRB, which blocks polymerase II dependent transcription (16). After DRB treatment, CypB-Nucl was still observed in spots inside the nucleus (Fig. 3A and D), which had a size and pattern typical for nuclear bodies. Given that Cajal bodies are known to be enriched in small nucleolar ribonucleoproteins, we performed an immunostaining for coilin, the major component of Cajal bodies. A strong colocalization with CypB-Nucl was detected in DRB treated cells. Colocalization was not complete; coilin spots were larger and enclosed CypB-Nucl. In contrast, no significant colocalization was found with other nuclear bodies such as PML bodies, as evidenced by immunostaining for PML (Fig. 3A). The colocalization observed between CypB-Nucl and RNA POL and UBF persisted upon disruption of nucleoli even after 400 min of DRB treatment. Fibrillarin, also encapsulated the CypB spots, similar to coilin (Fig. 3D).

In silico analysis reveals potential interacting partners involved in ribosome biogenesis and RNA processing. Sparked by our observations from the deletion screen we wondered whether СypB could have a role in nucleolar functions. To this end we screened for potential nucleolar interaction partners of CypB by combining information from two resources: a microarray analysis of CypB siRNA targeting the 3'-end region of the CypB coding sequence performed in a human 
cancer cell line (17) and secondly the Nucleolar Proteome Database (NoPdb), which contains over 700 nucleolar proteins detected by multiple mass spectrometry (18). A comparative analysis between two databases resulted in a list of 22 nuclear proteins that were regulated by $\mathrm{CypB}$ with a fold-change of more than 1.5 respective to the control (Table III). For validation, we selected two proteins [dyskerin (DCK1) and ISG20L2] for colocalization with CypB-Nucl. DCK1 was present in the nucleoli where it colocalized with CypB-Nucl. In addition, DCK1 was also found in the Cajal bodies as indicated by colocalization with coilin (Fig. 3D). However, treatment with DRB resulted in a pan-nuclear staining and no significant colocalization with CypB-Nucl. Surprisingly, ISG20L2 was not observed in the nucleoli. Instead ISG20L2 specifically localized to the cytoplasm in spots. As no colocalization was observed between CypB-Nucl and ISG20L2, we used the CypB-Full C-terminal construct to look for a potential interaction. When intense PDI spots were observed we always saw a strong colocalization with CypB-Full $\mathrm{C}$ as well as ISG20L2. Incubation with CsA reduced the presence of these intense spots (Fig. 3F). As previously described, a distinct subset (5\%) of the CypBFull $\mathrm{C}$ transfected cells resulted in a nuclear localization pattern similar to the one observed in CypB-Nucl. In these cells, PDI staining was found as a general nuclear staining. A small subset of the PDI remained in the cytoplasm in larger cytoplasmic bodies, where PDI and ISG20L2 were colocalized (Fig. 3F).

\section{Discussion}

In this report we describe the spatiotemporal behavior of CypB within human cell cultures. Immunofluorescent staining for CypB revealed two very distinct localization patterns: a cytoplasmic and a nuclear phenotype. Although $\mathrm{CypB}$ has been described in both the cytoplasm/ER (7) and the nucleus $(12,13)$ the exact regulatory pathway behind these different localization patterns remains unknown. In order to study the individual expression, we constructed two CypB-GFP constructs (CypB-Full and $\mathrm{CypB}-\mathrm{Nucl}$ ) that could reproduce the respective localization patterns observed for endogenously expressed CypB. These two constructs differ only in the first 33 amino acids. Possibly, a mechanism of alternative splicing mediates alternative localization of endogenous CypB. For efficient localization to the nucleus, CypB includes a nuclear localization sequence. DEKKKGPKVTVKV was previously proposed as an NLS signal (19).

In this study we revealed that nuclear CypB accumulates in the nucleoli, with -KGPKVTVKV- being the minimal NuLS signal. Based on colocalization studies with several nucleolar markers, we suggest that CypB-Nucl preferentially localizes in the fibrillar centers of the nucleoli. This is based on different observations as the spots specifically colocalized with RNA-POL, UBF and fibrillarin, which are known to reside within the fibrillar centers (20) and the lack of PI staining at the fibrillar centers. Fibrillarin has a potential RNA binding domain and is directly involved in many post-transcriptional processes including pre-rRNA processing, pre-rRNA methylation, and ribosome assembly $(21,22)$. UBF is an RNA-POL I factor essential for the activation of rDNA transcription by inducing remodeling of ribosomal gene chromatin (23) but also acts as a promoter of rDNA genes suggesting a role as activator of rDNA transcription (24). The accumulation of $\mathrm{UBF}$ and RNA-POL in the fibrillar centers is thought to function as a dynamic storage facility, where RNA-POL complexes are preassembled (20).

The exact function of $\mathrm{CypB}$ in the fibrillar centers remains unclear but it is tempting to presume a potential role in RNA processing or ribosome biogenesis. Our in silico analysis indicates that $\mathrm{CypB}$ regulates the expression of 22 nucleolar proteins. Approximately $40 \%$ of these proteins are known to be involved in transcription or RNA processing. One of these proteins DCK1, is a component of small nucleolar ribonucleoprotein complexes and modifies newly synthesized ribosomal, spliceosomal, and possibly other RNAs (25). In addition to its regulation by CypB, DCK1 colocalizes with CypB in the fibrillar centers and in the Cajal bodies, which indicates a continuous exchange and possible interaction in both subsections of the nucleus. It is interesting that $\mathrm{CypB}$ is an essential component for $\mathrm{HCV}$ replication as it interacts directly with RNA polymerase NS5B to stimulate its RNA binding activity $(9,11)$. The HCV virus hijacks and utilizes CypB to instigate its own viral RNA replication. HCV replication occurs at the cytoplasmic side of the ER but other viruses specifically migrate to the nucleolus where they colocalize with factors, such as nucleolin and fibrillarin (26). As CypB is present at both locations it is possible that other viruses also misuse CypB for their replication. It is plausible that $\mathrm{CypB}$ performs a similar endogenous function in the nucleus of mammalian cells and that it forms a complex with RNA-POL to increase RNA transcription efficiency.

Our immunofluorescence data revealed a clear cell cycle dependent pattern for $\mathrm{CypB}$ in human fibroblasts. CypB aggregated into foci that colocalized with the nucleoli and was clearly visible in the $\mathrm{S}$ and $\mathrm{G} 2$ phases. The activity of nucleoli depends on cell cycle regulators, with rDNA transcription reaching a maximum in the $S$ and $G 2$ phases, silent in mitosis, and slowly recovering in the $\mathrm{G} 1$ phase (27). As CypB followed this cell cycle pattern it further alludes to a role in rDNA transcription. Moreover UBF, RNA-POL, fibrillarin and coilin spots colocalized with these spots, but also partly encapsulated the CypB-Nucl. Disruption of the nucleolar architecture with DRB caused Cajal bodies to encapsulate CypB. Even though nucleolar morphology and interaction of the different nucleolar components are rapidly disturbed $(28,29)$, CypB-Nucl, UBF, RNA-POL and fibrillarin spots remained colocalized suggestive of direct interactions. Perhaps more importantly, incubations with DRB show little, if any, inhibition of rDNA transcription (30), which makes it tempting to speculate that CypB is part of an RNA transcription complex which remains intact upon nucleolar disruption. In this view, the encapsulation with coilin and fibrillarin might be a cellular response to create an alternative but appropriate compartment where RNA transcription can continue. The number of Cajal bodies is determined by snRNP levels and RNA processing rates in the nucleus and as such Cajal body numbers can quickly respond to changes in transcription rates (31-34).

Although the particular functional significance of CypB in the fibrillar centers of nucleoli remains uncertain, our results clearly demonstrate that $\mathrm{CypB}$ localizes to the nucleoli in an ATP-dependent fashion during the more transcriptionally active phases of the cell cycle. It also regulates a subfraction 
of proteins involved in transcription or RNA processing. As the fibrillar centers may represent dynamic storage centers for ribosomal biogenesis, it is possible that CypB is necessary for RNA transcription in a manner resembling $\mathrm{HCV}$ replication.

\section{Acknowledgements}

This study was financially supported by the Ghent University (BOF). The authors would like to thank Dr A. Haegeman, Dr W. Grunewald, K. De Vusser and B. Cannoot for their technical assistance. Dr W.H. De Vos for the critical reading of the manuscript. The support of the Hercules Foundation to Professor E.J.M. Van Damme (project AUGE/013) is gratefully acknowledged.

\section{References}

1. Wang P and Heitman J: The cyclophilins. Genome Biol 6: 226, 2005.

2. Rycyzyn MA, Reilly SC, O'Malley K and Clevenger CV: Role of cyclophilin B in prolactin signal transduction and nuclear retrotranslocation. Mol Endocrinol 14: 1175-1186, 2000.

3. Arber S, Krause KH and Caroni P: s-cyclophilin is retained intracellularly via a unique $\mathrm{COOH}$-terminal sequence and colocalizes with the calcium storage protein calreticulin. J Cell Biol 116: 113-125, 1992.

4. Mariller C, Allain F, Kouach M and Spik G: Evidence that human milk isolated cyclophilin B corresponds to a truncated form. Biochim Biophys Acta 1293: 31-38, 1996.

5. Denys A, Allain F, Masy E, Dessaint JP and Spik G: Enhancing the effect of secreted cyclophilin B on immunosuppressive activity of cyclosporine. Transplantation 65: 1076-1084, 1998.

6. Price ER, Jin M, Lim D, Pati S, Walsh CT and McKeon FD Cyclophilin B trafficking through the secretory pathway is altered by binding of cyclosporin A. Proc Natl Acad Sci USA 91: 3931-3935, 1994

7. Le Hir M, Su Q, Weber L, Woerly G, Granelli-Piperno A and Ryffel B: In situ detection of cyclosporin A: evidence for nuclear localization of cyclosporine and cyclophilins. Lab Invest 73: 727-733, 1995

8. Horibe T, Yosho C, Okada S, et al: The chaperone activity of protein disulfide isomerase is affected by cyclophilin $\mathrm{B}$ and cyclosporin A in vitro. J Biochem 132: 401-407, 2002.

9. Watashi K, Ishii N, Hijikata M, et al: Cyclophilin B is a functional regulator of hepatitis $\mathrm{C}$ virus RNA polymerase. Mol Cell 19: 111-122, 2005.

10. Rice CM and You S: Treating hepatitis C: can you teach old dogs new tricks? Hepatology 42: 1455-1458, 2005.

11. Heck JA, Meng X and Frick DN: Cyclophilin B stimulates RNA synthesis by the HCV RNA dependent RNA polymerase. Biochem Pharmacol 77: 1173-1180, 2009.

12. Bauer K, Kretzschmar AK, Cvijic H, et al: Cyclophilins contribute to Stat 3 signaling and survival of multiple myeloma cells. Oncogene 28: 2784-2795, 2009.

13. Rycyzyn MA and Clevenger CV: The intranuclear prolactin/ cyclophilin B complex as a transcriptional inducer. Proc Natl Acad Sci USA 99: 6790-6795, 2002.

14. Seifried HE, Anderson DE, Fisher EI and Milner JA: A review of the interaction among dietary antioxidants and reactive oxygen species. J Nutr Biochem 18: 567-579, 2007.

15. Kremers GJ, Goedhart J, van Munster EB and Gadella TWJ: Cyan and yellow super fluorescent proteins with improved brightness, protein folding, and FRET Förster radius. Biochemistry 45: 6570-6580, 2006.
16. Haaf T and Ward DC: Inhibition of RNA polymerase II transcription causes chromatin decondensation, loss of nucleolar structure, and dispersion of chromosomal domains. Exp Cell Res 224: 163-173, 1996.

17. Fang F, Flegler AJ, Du P, Lin S and Clevenger CV: Expression of cyclophilin B is associated with malignant progression and regulation of genes implicated in the pathogenesis of breast cancer. Am J Pathol 174: 297-308, 2009.

18. Leung AK, Trinkle-Mulcahy L, Lam YW, Andersen JS, Mann M and Lamond AI: NOPdb: nucleolar proteome database. Nucleic Acids Res 34: D218-D220, 2006

19. Bukrinsky MI: Cyclophilins: unexpected messengers in intercellular communications. Trends Immunol 23: 323-325, 2002.

20. Casafont I, Bengoechea R, Navascues J, Pena E, Berciano MT and Lafarga M: The giant fibrillar center: a nucleolar structure enriched in upstream binding factor (UBF) that appears in transcriptionally more active sensory ganglia neurons. J Struct Biol 159: 451-461, 2007.

21. Aris JP and Blobel G: cDNA cloning and sequencing of human fibrillarin, a conserved nucleolar protein recognized by autoimmune antisera. Proc Natl Acad Sci USA 88: 931-935, 1991.

22. Tollervey D, Lehtonen H, Jansen R, Kern H and Hurt EC: Temperature-sensitive mutations demonstrate roles for yeast fibrillarin in pre-rRNA processing, pre-rRNA methylation, and ribosome assembly. Cell 72: 443-457, 1993.

23. Stefanovsky V, Langlois F, Gagnon-Kugler T, Rothblum LI and Moss T: Growth factor signaling regulates elongation of RNA polymerase I transcription in mammals via UBF phosphorylation and r-chromatin remodeling. Mol Cell 21: 629-639, 2006.

24. O'Sullivan AC, Sullivan GJ and McStay B: UBF binding in vivo is not restricted to regulatory sequences within the vertebrate ribosomal DNA repeat. Mol Cell Biol 22: 657-668, 2002.

25. Ge J, Rudnick DA, He J, et al: Dyskerin ablation in mouse liver inhibits rRNA processing and cell division. Mol Cell Biol 30: 413-422, 2010

26. Hiscox JA: The nucleolus - a gateway to viral infection? Arch Virol 147: 1077-1089, 2002.

27. Sirri V, Urcuqui I, Roussel P and Hernandez-Verdun D: Nucleolus: the fascinating nuclear body. Histochem Cell Biol 129: 13-31, 2008.

28. Scheer U, Hugle B, Hazan R and Rose KM: Drug-induced dispersal of transcribed rRNA genes and transcriptional products: immunolocalization and silver staining of different nucleolar components in rat cells treated with 5,6-dichloro-betaD-ribofuranosylbenzimidazole. J Cell Biol 99: 672-679, 1984.

29. Haaf T, Hayman DL and Schmid M: Quantitative determination of rDNA transcription units in vertebrate cells. Exp Cell Res 193 78-86, 1991.

30. Tamm I, Hand R and Caliguiri LA: Action of dichlorobenzimidazole riboside on RNA synthesis in L-929 and HeLa cells. J Cell Biol 69: 229-240, 1976.

31. Cioce $M$ and Lamond AI: Cajal bodies: a long history of discovery. Annu Rev Cell Dev Biol 21: 105-131, 2005.

32. Sleeman JE, Ajuh P and Lamond AI: snRNP protein expression enhances the formation of Cajal bodies containing p80-coilin and SMN. J Cell Sci 114: 4407-4419, 2001.

33. Lafarga M, Berciano MT, Garcia-Segura LM, Andres MA and Carmo-Fonseca M: Acute osmotic/stress stimuli induce a transient decrease of transcriptional activity in the neurosecretory neurons of supraoptic nuclei. J Neurocytol 27: 205-217, 1998.

34. Lemm I, Girard C, Kuhn AN, et al: Ongoing U snRNP biogenesis is required for the integrity of Cajal bodies. Mol Biol Cell 17: 3221-3231, 2006 